\title{
ESTUDIO DE INSECTICIDAS PARA EL CONTROL DE MOSCA BLANCA Bemisia tabaci Genn EN EL CULTIVO DE FRIJOL Phaseolus vulgaris L.*
}

\author{
José E. Mancía**, José C. Escobar**, Adán Hernández**, Julio Soto** y Santos P. Bonilla**
}

\begin{abstract}
RESUMEN
Se evaluaron nuevos plaguicidas para reducir las poblaciones de la mosca blanca (Bemisia tabaci Genn), vector del Mosaico Dorado del frijol (BGMV), en el Valle de Zapotitán y la Estación Experimental de San Andrés a $460 \mathrm{mnsm}$, durante la época seca a partir del 22 de diciembre de 1989 a mayo de 1990. Las variedades de frijol utilizadas fueron Rojo de Seda, Sangre de toro y CENTA Cuscatleco. Se utilizó un diseño experimental de bloques al azar, con 4 y 5 repeticiones, y un total de 4 ensayos. Se encontró en el primer y segundo ensayo que bifentrin 100 EC (60 g.i.a./ha) y fenpropatrin 375 FC (203 g.i.a./ha) fueron eficaces para el control de la mosca blanca, y redujeron la incidencia del mosaico dorado. Cuando B. tabaci no se controló eficazmente la incidencia de BGMV alcanzó un 100\% entre los 36 y 32 días después de la siembra, en las variedades susceptibles Rojo de Seda y Sangre de Toro. En los ensayos tres y cuatro se utilizaron además tratadores de semilla y suelo combinados con aspersiones al follaje. Los mejores rendimientos se produjeron con los tratamientos de carbosulfan + bifentrin $(1519,9 \mathrm{~kg} / \mathrm{ha})$, acephato + bifentrin $(1428 \mathrm{~kg} / \mathrm{ha})$ en la variedad resistente CENIA Cuscatleco, acephato + fenpropatrin (756,6 $\mathrm{kg} / \mathrm{ha})$, acephato + bifentrin $(725,1 \mathrm{~kg} / \mathrm{ha})$ y carbofuran + fenpropatrin (636,3 g/ha) en la variedad susceptible Rojo de Seda. De los productos evaluables, fenpropatrin $375 \mathrm{EC}$, fue el que mostró las poblaciones más bajas de huevos y ninfas de B. tabaci. Existió una correlación significativa entre la época de aparición de los síntomas de la enfermedad BGMV y la producción. El tratamiento acephato 95\% + bifentrin $100 \mathrm{EC}$ el mayor beneficio neto seguido por carbofuran $5 \% \mathrm{~g}$ + bifentrin $100 \mathrm{EC}$.
\end{abstract}

\begin{abstract}
Several trials were conducted to evaluate the effectiveness of new insecticides in controlling the white fly Bemisia tabaci Genn, which is the vector of the Bean Golden Mosaic Virus (BGMV), at the Zapotitlan Valley and at the San Andres Experimental Station at 460 masl, during the dry season, from December 22, 1989 to May, 1990. A complete randomized block experimental design with 4 and 5 replications was used. Four trials were planted with the Rojo de Seda, Sangre de Toro and CENIA-Cuscatleco bean varieties. In the first and second trials, the chemicals bifentrin 100 EC (60 g.ai./ha) and fenpropatrin 375 EC ( 203 g.ai/ha) were efficient in controlling the whitefly, reducing the incidence of BGMV. If B. tabaci Genn is not controlled efficiently and timely, the BGMV incidence reaches $100 \%$ between 36 and 32 days after planting (DAP) on the susceptible varieties Rojo de Seda y Sangre de Toro. Seed and soil treatments combined with foliage spraying were also used in the third and fourth trials. The treatments of carbosulpan + bifentrin $(1519.9 \mathrm{~kg} / \mathrm{ha})$ and acephate + bifentrin $(1428 \mathrm{~kg} / \mathrm{ha})$ produced the highest yields with the resistent variety CENIA- Cuscatleco. Acephate + fenpropatrin $(756.6 \mathrm{~kg} / \mathrm{ha})$, acephate + bifentrin $(725.1 \mathrm{~kg} / \mathrm{ha})$ and carbofuran + fenpropatrin $(636.3 \mathrm{~g} / \mathrm{ha})$ produced the highest yields with the susceptible variety Rojo de Seda. Of the tested products, fenpropatrin $375 \mathrm{EC}$ showed the lowest populations of eggs and nymphs of $B$. tabaci Genn. There was a significant correlation between production and the time when the disease symptoms appeared. The largest Net Benefit was obtained with acephate 95\% + bifentrin 100 EC treatment, followed by carbofuran $5 \%+$ bifentrin $100 \mathrm{EC}$.
\end{abstract}

\section{INTRODUCCIÓN}

La mosca blanca Bemisia tabaci Genn, es una plaga primaria del cultivo de frijol, no por los daños mecánicos que pueda ocasionarle al cultivo, sino porque es vector de la enfermedad conocida como Mosaico Dorado del frijol (BGMV), que se ha convertido en los últimos años en un factor limitante de la producción, obligando en muchos casos al agricultor a destruirse su cultivo.

A partir de 1988, se observó que los insecticidas tradicionales existentes en el mercado local de El
Salvador para el control de la mosca blanca, no mostraban la eficacia que en un principio se les había atribuido (Mancia et al. 1972, Mancia y Díaz 1974) incrementándose el problema. Entre los insecticidas que se dan más recientemente para el combate de la mosca blanca están, para aplicación al momento de la siembra el aldicarb, teofenax, carbofurán. Para aplicación al follaje theodam, metasystox, el metamidofus, el monocrotodos y el acefato (Gá1vez y Cárdenas, 1980, Schoonhoven y Cárdenas 1985).

Como resultado de esta problemática y por ser el

\footnotetext{
* Trabajo presentado en la XXXVII Reunión Anual del PCCMCA. Panamá. 1991.

** Técnicos e investigadores. CENIA-MAG, El Salvador.
} 
cultivo de frijol, básico en la dieta alimenticia del pueblo salvadoreño, se efectuó la presente investigación para seleccionar nuevos plaguicidas eficaces para reducir las poblaciones de B. tabaci y por consiguiente del BGMV.

\section{MATERIALES Y MÉTODOS}

Los trabajos se realizaron en el Valle de ZapotitIán y la Estación Experimental de San Andrés a $460 \mathrm{msnm}$; durante la época seca a partir del 22 de diciembre de 1989 a mayo de 1990. En un tipo de suelo franco arenoso. Las variedades de frijol utilizadas fueron Rojo de Seda, Sangre de Toro y CENTA Cuzcatleco. Se utilizó un diseño experimental de bloques al azar, con 4 y 5 repeticiones, y un total de 4 ensayos. La distancia de siembra fue de 0,6 m entre surcos y 0,10 m entre semillas. Cada parcela estuvo conformada por 10 surcos de $5 \mathrm{~m}$ de longitud. La evaluación de los plaguicidas se realizó con base a muestre os de adultos de mosca blanca, consistiendo la unidad muestral de 10 plantas tomadas al azar en cada uno de los tratamientos y contándose en forma visual el total de B. tabaci por planta. Además, se hicieron muestreos de huevos y ninfas de la mosca blanca en 5 plantas tomadas al azar por tratamiento. Asimismo, se determinó la incidencia de mosaico dorado, contando el total de plantas en los dos surcos centrales de cada parcela y el número de plantas con síntomas de BGMV para determinar el efecto de la enfermedad en el número de vainas por plantas y el número de granos por vaina en la producción, para lo cual se marcaron plantas con síntomas de BGMV según la fecha de aparición de éstos, através de cintas plásticas de diferente color, para cada fecha de marcaje.

Los ensayos primero y segundo se ubicaron en el cantón Ceiba Mocha, con la variedad Rojo de Seda y en el cantón Tigre, con la variedad Sangre de Toro respectivamente. Los tratamientos utilizados fueron: bifentrin $100 \mathrm{EC}, 60 \mathrm{~g}$ i.a./ha; carbosulfan $480 \mathrm{EC}, 600$ g.i.a./ha; butocarboxim 50 EC, 750 g.i.a./ha; acephato 95 Ps, $1,1 \mathrm{~kg}$ g.ia./ha; metamidophos $600 \mathrm{CS}, 857 \mathrm{~g}$ i.a./ha y el testigo absoluto; en el segundo ensayo, se agregó a los tratamientos anteriores fenpropatrin $375 \mathrm{EC}, 203 \mathrm{~g}$ i.a./ha; Los ensayos tercero y cuarto se realizaron en el cantón Flor Amarilla, utilizando las variedades de frijol" CENTA Cuzcatleco" y Rojo de Seda, respectivamente; y los tratamientos de suelo, semilla y follaje fueron los siguientes: carbosulfan 25 ST 750 g.i.a. $/ 100 \mathrm{~kg}$ de semilla; acephato 95 PS, 750 g.i.a. $/ 100 \mathrm{~kg}$ de semilla carbofuran $5 \% \mathrm{~g}, 2 \mathrm{~kg} / \mathrm{ha}$; carbosulfan $25 \mathrm{ST}+$ fenpropatrin; carbosulfan $25 \mathrm{ST}+$ bifentrin $100 \mathrm{EC}$, acephato 95 PS + fenpropatrin 375 EC; acephato 95 PS + bifentrin $100 \mathrm{EC}$; carbofuran $5 \% \mathrm{G}+$ fenpropatrin 375 EC; carbofurano 5\% + bifentrin 100 EC y el Testigo Absoluto.

\section{RESULTADOS Y DISCUSION}

En el primer ensayo y segundo ensayo, el bifentrin 100 EC, 60 g i.a. y fenpropatrin 375 EC, 203 g.i.a./ha, fueron eficaces para control de la mosca blanca (Cuadros 1 y 2), reduciéndose la incidencia de mosaico dorado (las aplicaciones de estos productos, se iniciaron a la emergencia del frijol). Cuando no se controla eficazmente yen el tiempo adecuado B. tabaci Genn; la incidencia del BGMV, alcanzó un porcentaje del $100 \%$, entre los 36 y 32 DOS (días después de siembra), en las variedades susceptibles Rojo de Seda y Sangre de Toro.

Cuadro 1. Comparación de medias de los tratamientos y su porcentaje con base en el número de adultos de mosca blanca Bemisia tabaci Genn por muestreo. Frijol rojo de seda. 24 horas después de la 1ra, 2da y 3era. aplicación. Cantón Ceiba Mocha. Zapotitán. Dpto. La libertad.

\begin{tabular}{|c|c|c|c|c|c|c|}
\hline $\begin{array}{l}\text { Ap1)cacion } \\
\text { Tratamentos }\end{array}$ & lera & & पारान & & 36 & \\
\hline & meora & Eficac & רeणाइ & Eficac & & Eficac \\
\hline 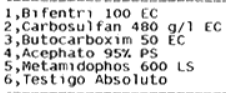 & 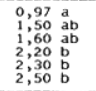 & $\begin{array}{l}91,00 \\
41,60 \\
25,30 \\
60,60 \\
50,00\end{array}$ & $\begin{array}{ll}0,8 & \mathrm{a} \\
1,8 & \mathrm{ab} \\
2,7 & \mathrm{~b} \\
2,4 & \mathrm{~b} \\
2,4 & \mathrm{~b} \\
2,8 & \mathrm{~b}\end{array}$ & $\begin{array}{l}92,00 \\
58 ; 00 \\
21 ; 00 \\
45 ; 00 \\
22,00\end{array}$ & $\begin{array}{l}1,8 \mathrm{a} \\
3,0 \mathrm{~b} \\
2,9 \mathrm{~b} \\
3,5 \mathrm{~b} \\
3,2 \mathrm{~b} \\
3,4 \mathrm{~b}\end{array}$ & $\begin{array}{l}89,50 \\
43,90 \\
37,70 \\
22,40 \\
15,50\end{array}$ \\
\hline c.v. & $18,60 \%$ & & $21,5 \%$ & & $19,2 \%$ & \\
\hline
\end{tabular}

Cuadro 2. Comparación de medias de tratamientos y su porcentaje de eficacia con base en el número de adultos de mosca blanca Bemisia tabaci Genn por muestreo. Frijol sangre de toro. 24 horas después de la 1era., 2da. y 3ra. aplicación. Cantón El Tigre, Zapotitán. Dpto. La Libertad.

\begin{tabular}{|c|c|c|c|c|c|}
\hline \multirow{2}{*}{$\begin{array}{l}\text { Aplicacion } \\
\text { Tratanientos }\end{array}$} & \multicolumn{2}{|c|}{ lera } & \multicolumn{2}{|c|}{2 da } & \multirow{2}{*}{ memd eficac } \\
\hline & & Efica & & Efic. & \\
\hline 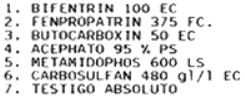 & $\begin{array}{ll}0.6 & \mathrm{a} \\
0.4 \mathrm{a} \\
11.6 \mathrm{~d} \\
2.2 \mathrm{~d} \\
3.2 \mathrm{~d} \\
3.6 \mathrm{a} \\
6.8 \mathrm{~d}\end{array}$ & $\begin{array}{l}84.8 \\
84.3 \\
70.4 \\
61: 9 \\
47.0 \\
36.0\end{array}$ & $\begin{array}{l}0.6 \mathrm{~d} \\
0.6 \mathrm{a} \\
17.4 \mathrm{~b} \\
19.6 \mathrm{~b} \\
19.2 \mathrm{~b} \\
14.4 \mathrm{bb} \\
26.6 \mathrm{~b}\end{array}$ & $\begin{array}{l}97.00 \\
93.00 \\
388.70 \\
48.00 \\
41: 70 \\
56.90\end{array}$ & $\begin{array}{lll}0.6 & \mathrm{a} & 90.3 \\
0.6 & \mathrm{a} & 80.3 \\
11.8 & \mathrm{ab} & 70.5 \\
36.2 \mathrm{~b} & 36.1 \\
33.4 & \mathrm{~b} & 36.4 \\
22.2 & \mathrm{ab} & 23.6 \\
37.8 & \mathrm{~b} & -6\end{array}$ \\
\hline c.v. & $63.3 \%$ & & $55.8 \%$ & & $67.9 \%$ \\
\hline
\end{tabular}

Cuando se evaluó la efectividad del tratamiento a la semilla y suelo, combinados con aspersiones al follaje (ensayos 3 y 4), la mejor eficacia en el control de mosca blanca se obtuvo cuando se realizaron tratamientos al follaje (Cuadros 3 y 4) y los mejores rendimientos se encontraron con carbosulfan + bifentrin, 1519,9 (t10) $\mathrm{kg} / \mathrm{ha}$; acephato + bifentrin, 1428 (t8) $\mathrm{kg} / \mathrm{ha}$ en la variedad resistente CENTA Cuzcatleco y acephato + fenpropatrin, $751,6 \mathrm{a}, \mathrm{kg} / \mathrm{ha}$ (t7) acephato + bifentrin 725,1 (t8) kg/ha y 
carbofuran + fenpropatrin, 636,3 (t9) g/ha, en la variedad susceptible Rojo de Seda. Asimismo, se encontró que cuando los síntomas de la enfermedad aparecían a los 17 DDS, la variedad Rojo de Seda tuvo un promedio de vainas por planta de 0,5 y la variedad resistente CENT A Cuscatleco, 1,7; a medida que los síntomas aparecían e iban acercándose a la floración, el número de vainas aumentaba, así en la variedad susceptible Rojo de Seda a los 44 DDS, se tuvo un promedio de 6.6 vainas por planta y en CENTA Cuscatleco de 10 a los 46 DDS ; lo mismo sucedió con el número de granos por vainas cuando los síntomas aparecieron a los 16 DDS, Rojo de Seda tuvo un promedio de 0,6 granos por vainas, y CENTA Cuscatleco 1,7, con síntomas a los 44 DDS, Rojo de Seda presentó una media de 3,1 granos por vaina y 4,3 granos por vaina CENTA Cuscatleco a los 46 DDS.

Cuadro 3. Comparación de medias de los tratamientos con base en el número de adultos de Bemisia tabaci Genn por muestreo. Frijol Centa Cuscatleco. 24 horas despúes de la 1era.,2da. y 3ra. aplicación. Flor Amarilla. San Andrés. Dpto La Libertad. 1990.

\begin{tabular}{|c|c|c|c|c|}
\hline TRATAMIENTOS & $\mathrm{X}$ & $\mathrm{X}$ & $\mathrm{X}$ & \\
\hline 1. Testigo Absoluto & $64.5 \mathrm{~b}$ & $124.0 \mathrm{c}$ & 128.5 & c \\
\hline 2. Carbosulfan $25 \mathrm{ST}$ & $10.8 \mathrm{a}$ & $74.0 \mathrm{~b}$ & 49.0 & $\mathrm{~b}$ \\
\hline 3. Acephato $95 \%$ PS & $9.0 \mathrm{a}$ & $75.8 \mathrm{~b}$ & 20.5 & $\mathrm{~b}$ \\
\hline 4. Carbofurano & $10.0 \mathrm{a}$ & $73.3 \mathrm{~b}$ & 43.0 & $\mathrm{~b}$ \\
\hline \multicolumn{5}{|l|}{ 5. Carbosulfan $25 \mathrm{ST}+$} \\
\hline Fenpropatrin 375 EC & $0.5 \mathrm{a}$ & $0.5 \mathrm{a}$ & 0.3 & a \\
\hline \multicolumn{5}{|l|}{ 6. Carbosulfan $25 \mathrm{ST}+$} \\
\hline Bifentrin 100 EC & $0.5 \mathrm{a}$ & $0.8 \mathrm{a}$ & 0.3 & \\
\hline \multicolumn{5}{|l|}{ 7. Acephato $95 \mathrm{PS}+$} \\
\hline Fenpropatrin 375 EC & $0.3 \mathrm{a}$ & $0.5 \mathrm{a}$ & 0.3 & \\
\hline \multicolumn{5}{|l|}{ 8. Acephato 95 PS } \\
\hline Bifentrin 100 EC & $0.5 \mathrm{a}$ & $0.5 \mathrm{a}$ & 0.3 & \\
\hline \multicolumn{5}{|l|}{ 9. Carbofurano $5 \% \mathrm{G}+$} \\
\hline Fenpropatrin 375 EC & $0.5 \mathrm{a}$ & $0.5 \mathrm{a}$ & 0.3 & \\
\hline \multicolumn{5}{|l|}{ 10. Carbofurano $5 \% \mathrm{G}+$} \\
\hline Bifentrin 100 EC & $0.5 \mathrm{a}$ & $0.8 \mathrm{a}$ & 0.5 & \\
\hline
\end{tabular}

Se la relacionó negativamente la época. de aparición de los síntomas de la enfermedad entre los 17 y 44 DDS, con la producción, la que varió de $30 \mathrm{~kg}$ a 1013,2 kg/ha para Rojo de Seda y de $130 \mathrm{~kg}$ a 1339,6 kg para CENTA Cuscatleco. Las plantas que presentaron síntomas posteriores a esta época, produjeron 1274,3 kg/ha en Rojo de Seda y $1791,5 \mathrm{~kg} / \mathrm{ha}$ en CENTA Cuscatleco.
De todos los productos evaluados fenpropatrin 375 $\mathrm{EC}$, fue el que mostró las poblaciones más bajas de huevos y ninfas de $B$. tabaci.

Cuadro 4. Comparación de medias de los tratamientos con base en el número de adultos de Bemisia tabaci Genn por muestreo. Frijol rojo de seda. 24 horas despúes de la 1era.,2da. y 3ra. aplicación. Flor Amarilla. San Andrés. Dpto. La Libertad. 1990

\begin{tabular}{|c|c|c|c|}
\hline TRATAMIENTOS & $\mathrm{X}$ & $\mathrm{x}$ & $\mathrm{x}$ \\
\hline 1. Testigo Absoluto & $44.3 \mathrm{~b}$ & $62.3 \mathrm{c}$ & $113.0 \mathrm{~b}$ \\
\hline 2. Carbosulfan $25 \mathrm{ST}$ & $48.3 \mathrm{~b}$ & $44.0 \mathrm{bc}$ & $90.8 \mathrm{~b}$ \\
\hline 3. Acephato $95 \%$ PS & $64.5 \mathrm{~b}$ & $29.8 \mathrm{bc}$ & $74.5 \mathrm{~b}$ \\
\hline 4. Carbofurano & $49.5 \mathrm{~b}$ & $24.3 \mathrm{ab}$ & $109.3 \mathrm{~b}$ \\
\hline 5. Carbosulfan $25 \mathrm{ST}+$ & & & \\
\hline Fenpropatrin 375 EC & $0.5 \mathrm{a}$ & $0.8 \mathrm{a}$ & $1.3 \mathrm{a}$ \\
\hline 6. Carbosulfan 25 ST + & & & \\
\hline Bifentrin $100 \mathrm{EC}$ & $0.8 \mathrm{a}$ & $1.0 \mathrm{a}$ & $0.3 \mathrm{a}$ \\
\hline 7. Acephato 95 PS + & & & \\
\hline Fenpropatrin 375 EC & $0.0 \mathrm{a}$ & $0.3 \mathrm{a}$ & $1.0 \mathrm{a}$ \\
\hline 8. Acephato 95 PS & & & \\
\hline Bifentrin $100 \mathrm{EC}$ & 0.8 a & $1.0 \mathrm{a}$ & $1.3 \mathrm{a}$ \\
\hline 9. Carbofurano $5 \% \mathrm{G}+$ & & & \\
\hline Fenpropatrin 375 EC & $0.5 \mathrm{a}$ & $0.8 \mathrm{a}$ & $0.8 a$ \\
\hline 10. Carbofurano $5 \% \mathrm{G}+$ & & & \\
\hline Bifentrin 100 EC & $1.0 \mathrm{a}$ & $1.3 \mathrm{a}$ & $1.5 \mathrm{a}$ \\
\hline
\end{tabular}

Se realizó un análisis económico al ensayo 3, en el cual se utilizó la variedad de frijol resistente CENTA Cuscatleco. El análisis económico indica que el tratamiento acephato $95 \%$ + bifentrin, $100 \mathrm{EC}$, que tuvo mayor beneficio neto 4281,4 colones/ha, seguido de carbofuran 5\% g + bifentrin $100 \mathrm{EC}$, con 4121,8 colones/ha. El análisis de dominancia indica que el tratamiento carbofuran $5 \% \mathrm{~g}+$ fenpropatrin $375 \mathrm{EC}$, fue dominado, ya que tuvo el mayor total de costos 1431,3 colones/ha y el menor beneficio neto 3976 colones/ha que el tratamiento acephato 95\% PS + fenpropatrin $375 \mathrm{EC}$, que tuvo 891,8 colones/ha del total de costos y 3987,4 colones/ha de beneficio neto.

$\mathrm{El}$ análisis marginal indica que el tratamiento acephato 95\% PS + fenpropatrin tuvo un beneficio neto adicional o marginal de 872 , 2/ha y una tasa de retorno marginal del $98 \%$, o sea, que por cada colón invertido en este tratamiento, se recuperó 1,98 colones.

\section{CONCLUSIONES}

El tratamiento carbofuran 5\% $8+$ bifentrin $\mathrm{EC}+$ fenpropatrin $375 \mathrm{EC}$, fueron eficaces para el control de $B$. tabaci Genn y reducir la incidencia del BGMV.

El control del vector del mosaico dorado, debe iniciarse a la emergencia del frijol para tener éxito en la disminución de la enfermedad. 
Existió correlación significativa entre la época de aparecimiento de los síntomas de la enfermedad (BGMV) y la producción.

El tratamiento acephato $95 \%$ PS + fenpropatrin, tuvo una tasa de retorno marginal del $98 \%$.

\section{LITERATURA CITADA}

GALVEZ, O.B.; CARDENAS, M.R. 1980. Virus transmitidos por moscas blancas. In Problemas de producción del frijol: enfermedades, insectos, limitaciones edáficas y climáticas de Phaseolus vulgaris L. Schwartzy Gálvez eds. Cali, Colombia. CIAT. pp. 261-274.
MANCIA, J. E.; DIAZ A. DE J.; SMITH F. 1972. Insecticidas sistémicos para control de mosca blanca Bemisia tabaci Genn e infección virosa en el frijol. In XVIII Reunión Anual del PCCMCA, Managua, Nicaragua. Actas de la mesa de leguminosas de grano IICA, Zona Norte. pp. 110114.

MANCIA J. B.; DIAZ. CH. A DE J. 1974. Utilización de insecticidas sistémicos granulados en el control de mosca blanca Bemisia tabaci Genn. e información virosa en el frijol común. SIADES (El Salvador) 3 (3): 77-81.

SOONHOVEN, AV.; CARDONA, C. 1985. Plagas que atacan el follaje. In Frijol: investigación y producción. Cali, Colombia. CIAT. pp. 270-272. 